\title{
Field Evaporation Behavior of Metal Oxide/Metal Interfaces
}

\author{
Mukesh Bachhav ${ }^{1}$, Francois Vurpillot ${ }^{2}$, Frederic Danoix ${ }^{2}$, Raphaële Danoix ${ }^{2}$, Beatrice Hannoyer ${ }^{2}$, \\ Emmanuelle A. Marquis ${ }^{1}$ \\ ${ }^{1 .}$ Department of Materials Science and Engineering, University of Michigan, Ann Arbor, MI, USA \\ 2. Groupe de Physique des Matériaux, Université et INSA de Rouen - UMR CNRS 6634 - Normandie \\ Université, 76801 St Etienne du Rouvray, France
}

Thin films of metal oxides (MO) grown/deposited on metals ( $\mathrm{M}^{\prime}$ ) and semiconductors find its application in wide domain of research such as giant magneto resistance, tunnel barrier based devices, coatings for surface treatment, resistive switching, dilute magnetic semiconductors. Precise knowledge on the composition of metal oxides and across the interface of MO-M' is extremely important for their functional properties, applicability and also to understand the underlying physics. Thus interfacial study on metal oxide-metal is important not only for material science research (understanding oxidation mechanism of metals/alloys) but also from technological point of view for devices (resistive switching in transition metal oxide). Among all the analytical techniques for elucidating chemistry and 3D distribution at small length scale, atom-probe tomography (APT) has already demonstrated its capability to analyze materials with poor conductivity, such as dielectrics, ceramics, metal oxides and semiconductors.

The analysis of metal oxide/metal system is however more challenging. Differences in the evaporation fields of metals and metal oxides often introduce artifacts in the 3D reconstruction of the data [1]. Apart from reconstruction limitations, the presence of an apparent additional metal oxide layer at the interface of the metal and the metal oxide is often observed when the oxide phase is on top or next to the metal phase within the APT specimen [2]. While this oxide layer may be formed in some MO-M' systems, it may also be an artifact of field evaporation. Marquis et al. showed that the apparent interfacial oxide layer did not appear when preparing samples with the metal phase on top of the oxide phase [2]. A number of examples can be found in the literature for a wide range of MO-M' system and interfacial geometries, where the presence of the interfacial layer could lead to misinterpretation.

The case where $M$ and $M^{\prime}$ are the same metal is not an expectation. Figure 1 shows the evaporation of $\mathrm{Fe}_{2} \mathrm{O}^{2+}$ ions at the metal/oxide interface. A likely mechanism is the migration of oxygen ions under the electric field present in the metal oxide. The ions migrate towards metal electrode and field evaporates with the metal atoms to form an apparent metal oxide layer. This behavior is analogous to oxygen migration in resistive switching devices during forward bias [3], and could provide an indirect observation of the resistive switching mechanism. [4]

\section{References:}

[1] C. Oberdorfer and G. Schmitz, Microscopy and Microanalysis 17, (2011) 15.

[2] E. Marquis et al, Journal of microscopy 241, (2011), 225.

[3] A Sawa, Materials today 11, (2008), 28.

[4] This work was supported by the University of Michigan College of Engineering and the Michigan Center for Materials Characterization. 


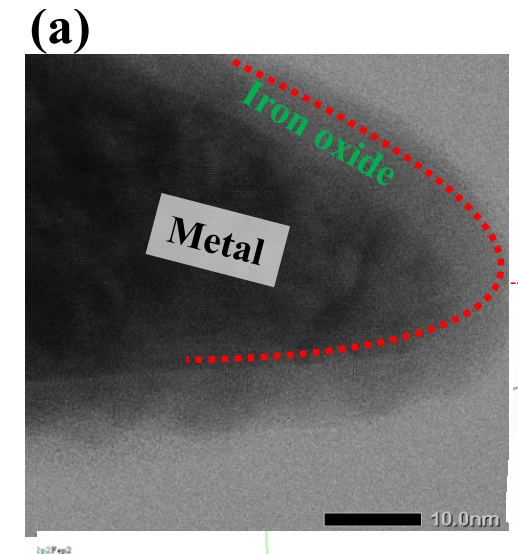

(b)
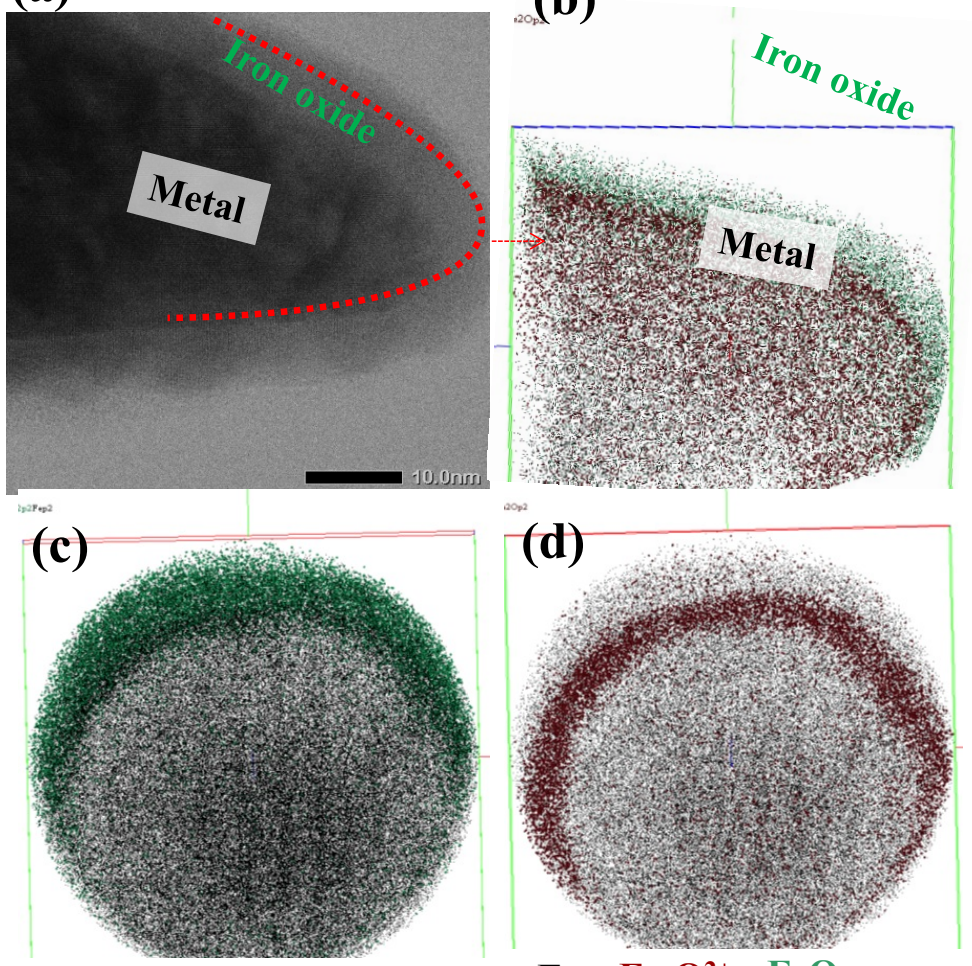

$\mathrm{Fe} \quad \mathrm{Fe}_{2} \mathrm{O}^{2+} \mathrm{FeO}$

Figure 1 (a) TEM image of tip of iron oxide grown on top of iron (b) 3-D reconstruction of a tip shown in (a). (c)\&(d) Distribution of atom/ions $\left(\mathrm{FeO}, \mathrm{Fe}\right.$ and $\left.\mathrm{Fe}_{2} \mathrm{O}^{2+}\right)$ in the view of detector. 